\title{
Using Video Modeling to Teach a Meal Preparation Task to Individuals with a Moderate Intellectual Disability
}

\author{
Philip M. Kanfush (D) and Jordan W. Jaffe \\ Saint Vincent College, Latrobe, PA, USA \\ Correspondence should be addressed to Philip M. Kanfush; philip.kanfush@stvincent.edu
}

Received 21 August 2018; Accepted 10 February 2019; Published 3 March 2019

Academic Editor: Gwo-Jen Hwang

Copyright (c) 2019 Philip M. Kanfush and Jordan W. Jaffe. This is an open access article distributed under the Creative Commons Attribution License, which permits unrestricted use, distribution, and reproduction in any medium, provided the original work is properly cited.

\begin{abstract}
A single-subject study implementing an $\mathrm{AB}$ design with 3 replications was conducted with 4 adolescents having multiple disabilities, including moderate cognitive impairments, to demonstrate the efficacy of a video modeling and video-prompting intervention to teach a food preparation skill. Each participant was taught to prepare a different food item using a task analysis comprising 50 to 64 steps. Within 12 training sessions, each participant achieved criterion performance, completing at least $90 \%$ of their cooking task steps independently. Three out of 4 participants maintained their food preparation skill in a maintenance probe taken 6 weeks after instruction ended. The fourth participant completed $89 \%$ of the task's steps independently during the maintenance probe. The findings of this study are consistent with those of earlier studies and suggest that video modeling may be a very effective and efficient method for promoting independence, participation, and self-determination among individuals with moderate intellectual disabilities. This study extends the literature base by focusing on longer, more complex meal preparation tasks than earlier studies.
\end{abstract}

\section{Introduction}

A significant component of quality of life is independence. In special education programs, independence is promoted through instruction in skills like choice-making, decisionmaking, and problem-solving [1]. One daily living activity that utilizes each of these skills is food preparation. The ability to handle, prepare, and store food safely is a critical independent living skill for young adults with intellectual disabilities [2]. In addition to fostering independent living, learning cooking skills has been noted to promote independence, dignity, and self-determination in individuals with disabilities [3]. Where these skills are lacking, limitations in functional independence can curtail an individual's participation in home and community settings, reduce his/her freedom of choice, and adversely impact postsecondary outcomes. Consequently, it is important for individuals with moderate and severe disabilities to learn meal preparation skills.

A variety of strategies have been used to teach cooking skills to individuals with intellectual disabilities, including picture prompts $[4,5]$, simultaneous prompting [6], and
Internet websites [3]. However, the literature suggests that one very effective method may be video modeling.

Video modeling is an instructional technique based on the principles of observational learning. Miltenberger and Charlop [7] note that "with video modeling, the child watches videotaped clips of the targeted behavior(s) being modeled" (p. 341). The child is then provided with opportunities to engage in the modeled behavior(s). Though all forms of video modeling follow this same general process, many variations exist. The simplest and most common variation is basic video modeling. In basic video modeling, all steps in the target task, skill, or behavior are presented to the participant. Then, the participant is given an opportunity to perform the target task, skill, or behavior. Another variation is video prompting, which Kellems and Edwards [8] define as "a form of video modeling in which the video is broken down into different clips or segments. The individual watches a single clip of a step in a task and is then prompted to perform the step shown" (p. 207).

A growing body of literature suggests that video modeling may be an effective instructional technique for teaching meal preparation skills to individuals with moderate or 
severe disabilities. For example, Al-Salahat [9] used video modeling to teach three young men with Down syndrome how to make a cream cheese sandwich. Each of the participants reached criterion, performing $100 \%$ of the task steps correctly across three consecutive intervention probes, generalized the skills, and maintained them 14 days later. The researchers concluded that basic "video modeling is an effective strategy in educating the pupils with intellectual disabilities the skill to prepare a simple meal" (p. 88) and that learning through video modeling provides students with training opportunities on many different skills before exposure to them during their lives" (p. 88). Johnson et al. [10] evaluated the effectiveness video prompting using an iPod touch to teach food preparation skills to two adolescents with autism. Their results demonstrated that video prompting was effective in enhancing the students' independent performance of the task. Ayres and Cihak [11] evaluated the effectiveness of computer-based video instruction for teaching meal-related skills. Working with three students with intellectual disabilities, they used computer-based video instruction to make a sandwich, operate a microwave oven, and set a table for a meal. Their findings indicated that while each of the students showed growth in the percentage of task steps completed correctly, a subtle decline in accuracy was observed during the maintenance phase of the study. However, each student returned to former performance rates after just one follow-up session of the intervention. This suggests that video modeling may be effective both for initial instruction and subsequent remediation.

Mechling and Gustafson [5] evaluated the effectiveness of video prompting in comparison to the presentation of static picture prompts in teaching 20 discrete cooking-related tasks to six adolescent males (ages 15 to 21) diagnosed with mild to moderate autism. Some of the tasks included cracking two eggs open into a bowl, turning an oven dial to bake at $350^{\circ}$, peeling a carrot with a vegetable peeler, and measuring $1 / 4$ cup of water from the sink. Mechling and Gustafson found that all six of their participants consistently improved their rates of task completion under the video-prompting condition as compared to static picture prompting. While only two of their six participants attained perfect performance of all 20 tasks using video prompting, all of the students showed substantial improvement over baseline performance levels, surpassing their average task completion in the static picture prompting condition by nearly double.

Later, Mechling and Gustafson [4] conducted a replication study with six high school-aged individuals (three females and three males) diagnosed with moderate intellectual disabilities and found strikingly similar results. All six participants substantially increased the percentage of steps they completed correctly over baseline using video prompting. The average percentage of tasks performed correctly across the six students using video prompting was nearly double that of the static pictures intervention (p. 112).

The research evidence suggests that video modeling and prompting may be effective instructional techniques for teaching meal preparation tasks to adolescents and adults with varied disabilities. However, these individuals form a very diverse group of people with different patterns of strengths, needs, and preferences. The present study adds to the literature by evaluating the efficacy of a combination of basic video modeling and video prompting to teach a food preparation task to four teenagers with multiple disabilities, including moderate intellectual disability.

\section{Method}

2.1. Participants and Setting. The study was conducted at a private, state-licensed school for exceptional children located in the Commonwealth of Pennsylvania. Four students between the ages of 13 and 17 were identified by school staff to participate in the study. All of the students had multiple disabilities and were identified based upon the criteria that they have a moderate cognitive impairment, a transition goal related to cooking specified in their individualized educational plans, no prior exposure to task analytic or video modeling instructional strategies, and the ability, in the opinion of the school staff, to attend to a video and imitate it. Their varying functioning levels, communication skills, and adaptive behaviors prevented each of the students from participating in the general education curriculum.

Cody, aged 15, was diagnosed with Down syndrome, a moderate intellectual disability, hearing and vision impairments, asthma, and a speech articulation disorder. Zeke, aged 15, also was diagnosed with Down syndrome, a moderate intellectual disability, bilateral hearing impairment corrected by hearing aids, and vision impairment. Additionally, Zeke was being treated for attention deficithyperactivity disorder. Lisa, aged 17 , had Down syndrome with a moderate cognitive disability and vision impairment. Her speech articulation was significantly impaired. The fourth student, Daniel, was a 13-year-old with moderate intellectual disability coupled with fine and gross motor impairments and self-stimulatory behaviors. All four students were placed in life skills support classrooms, where they benefited from direct instruction in functional academics, including reading and math.

All baseline sessions and training sessions were conducted in the "Domestic Arts" room at the school. The room contained four workstations, each having various utensils, cookware, and an electric stove/oven. Additionally, each workstation was equipped with a microwave oven. There was one sink shared between each two workstations and a common refrigerator shared by all four workstations. A bank of cabinets along two walls housed a variety of containers, measuring implements, flatware, and dishware shared by the four workstations. Additionally, the workstations shared a common garbage can and recycling bin. For Cody, two training sessions took place in his home kitchen to accommodate scheduling conflicts.

2.2. Target Behaviors and Task Analyses. Each student was taught a different cooking skill. The researchers asked the mothers of the participants to identify cooking skills that they deemed to be beneficial in the context of their home lives and children's preferences. Cody's mother selected pizza-making, while Zeke's mother chose cooking pasta. Lisa 
was taught to scramble eggs, and Daniel learned to make spaghetti. These tasks ranged in complexity from 50 steps for scrambling eggs to 64 steps for making pizza. An instructional objective for each student was established with a minimum criterion of $90 \%$ independent performance of all task steps across three consecutive sessions. The task analysis for pasta making is shown in Figure 1.

\section{Materials}

Each student was provided with the requisite ingredients, cookware, and utensils needed to prepare the assigned food item. Electric stoves/ovens were used to cook the food items.

Additionally, for each task, a video DVD was prepared in which a typically developing adult served as the model. Only one of the videos was filmed in the Domestic Arts classroom where instructional sessions were conducted. The other three videos were filmed in the models' homes. Each of the videos, which depicted the model preparing the food item and narrating each step of the procedure, was filmed using a Flipcam ${ }^{\circledR}$ video recorder and edited into DVD form using iMovie $^{\circledR}$ and $\mathrm{iDVD}^{\circledR}$ software. Chapter markers were inserted into the videos during the editing process to facilitate locating specific cooking steps in the videos quickly during instruction.

During instructional sessions, each student was provided with a portable DVD player and earphones for viewing the video and minimizing ambient distracting noises.

3.1. Experimental Design. A single-subject $\mathrm{AB}$ design with three replications was implemented. After three baseline probes with each student, the video modeling intervention was implemented for twelve training sessions. Data were collected during each baseline probe and each training session by graduate students working in independent teams with each student.

\subsection{Procedure}

3.2.1. Baseline. Each participant's ability to complete their respective cooking tasks was assessed during three baseline probes. At the beginning of each baseline probe, the participant wasgiven the instructional cue: "Let's make (food item)." No additional prompting or feedback was provided during these sessions, which were filmed to permit data reliability assessment. As the participant performed the task, the researcher recorded on a data sheet each step as it was completed. The probe was discontinued at the initial point at which the student deviated from the task analysis. The percentage of steps completed correctly was divided by the total number of steps in the task to determine the students' baseline completion rates.

3.2.2. Intervention. The procedures used during the instructional sessions are illustrated in flow chart form in Figure 2. Each instructional session began with a probe in which the student was given a single cue to prepare the required food item. The instructor recorded each step that the participant performed independently and correctly on a data collection sheet. No additional prompting or reinforcement was provided during the probe.

When the participant made his/her first error, the probe was stopped immediately, and a notation was made to mark the step on the data collection sheet where the probe ended (where the error occurred). The participant was then directed to the portable DVD player, where they were shown the video in its entirety. This occurred only one time during each session and comprised the video modeling component of the intervention. It was believed that showing the video once completely through would provide context for the video prompting to follow.

After the participant viewed the whole video, the scene selection menu was used to show the participant that step (and only that step) on which he/she made the error that ended the probe (video prompting). The instructor then gave them a verbal prompt to complete the modeled step. This prompt was kept purposely vague, such as "Go!" to prevent introducing an additional level of prompting as a confounding variable.

If the participant correctly completed the step, specific verbal praise was given (e.g., "Good job getting the sauce out of the refrigerator!"). The instructor then waited a latency period of five seconds to allow the participant to continue the behavior chain on their own.

Video prompting was implemented under two conditions. First, when the participant failed to initiate the next step in the behavior chain within the five second latency, the instructor directed him/her back to the video with the verbal prompt, "Look at what you need to do next," but limited his/ her view to the next step in the chain. The instructor then redirected the student back to the task as indicated above.

Second, if the participant began but completed a step incorrectly, the instructor interrupted the student and redirected him/her to the DVD player saying, "Look at how the person in the video did it." The participant were then given the vague verbal prompt to return to the task after the clip was replayed.

Care was taken throughout implementation of the intervention to avoid the introduction of extraneous verbal or gestural prompts.

3.3. Measures. Data were collected during each instructional session with the instructor noting which steps the participant completed independently and which steps they completed with prompts. At the conclusion of each instructional session, the instructor calculated the percentage of steps that the participant completed independently by dividing the total number of steps completed independently by the total number of task steps and then multiplying by 100 . The instructor also calculated the percentage of steps that the participant completed with prompting. Criterion was defined as completing $90 \%$ or more of the task steps independently across three consecutive training sessions.

\subsection{Reliability}

3.4.1. Interobserver Agreement. Data were validated for each student by calculating interobserver agreement. Cooper et al. 


\begin{tabular}{|c|c|}
\hline 1. Wash hands. & $\begin{array}{l}\text { 28. Lift wooden spoon and stir noodles slowly and carefully } \\
\text { then place wooden spoon back down on counter. }\end{array}$ \\
\hline $\begin{array}{l}\text { 2. Check supplies: pot, one cup measuring cup, strainer, wooden } \\
\text { spoon, microwave safe bowl, oven mitts, plate, fork. }\end{array}$ & 29. Locate and walk to get oven mitts on counter. \\
\hline 3. Check food supplies: pasta, sauce, parmesan cheese. & 30. Orient to microwave. \\
\hline 4. Locate pot, carry to kitchen sink, and place pot into sink. & 31. Put on oven mitts to protect hands from hot bowl. \\
\hline $\begin{array}{l}\text { 5. Turn cold water handle on, fill pot up to no more than } \\
\text { halfway with water, then turn faucet off. }\end{array}$ & 32. When microwave timer goes off, open microwave door. \\
\hline $\begin{array}{l}\text { 6. Lift pot with both hands out of sink, walk pot carefully } \\
\text { over to stove, and place carefully onto right front burner. }\end{array}$ & $\begin{array}{l}\text { 33. Reach in carefully with both hands and take out } \\
\text { microwave safe bowl. }\end{array}$ \\
\hline $\begin{array}{l}\text { 7. Reach to last knob on right of stove and push knob in then } \\
\text { turn to right until set on high. }\end{array}$ & $\begin{array}{l}\text { 34. With both hands, carefully carry microwave safe bowl to } \\
\text { counter and set down next to plate and fork. }\end{array}$ \\
\hline 8. Watch for bubbles to appear in water. & 35. Take off oven mitts and set on counter. \\
\hline $\begin{array}{l}\text { 9. While waiting for bubbles to appear, walk to pasta and } \\
\text { open the container. }\end{array}$ & 36. Remove the paper towel from top of bowl. \\
\hline 10. Locate and bring measuring cup to the pasta. & 37. Walk back and close microwave door. \\
\hline $\begin{array}{l}\text { 11. Using both hands, pick up and carefully pour pasta in measuring } \\
\text { cup until full. }\end{array}$ & 38. Walk to counter and get strainer. \\
\hline $\begin{array}{l}\text { 12. Take measuring cup of pasta over to pot with water on } \\
\text { stove and sit cup of pasta beside stove on counter. }\end{array}$ & 39. Walk to kitchen sink and set down in sink. \\
\hline $\begin{array}{l}\text { 13. If boiling then slowly and carefully pour pasta into } \\
\text { boiling water being careful not to touch the hot pot. }\end{array}$ & 40. When stove timer goes off, walk to stove. \\
\hline $\begin{array}{l}\text { 14. Get spoon then slowly and carefully stir pasta. After stirring set } \\
\text { spoon down next to stove. }\end{array}$ & 41. Turn off timer by pressing cancel. \\
\hline $\begin{array}{l}\text { 15. Set timer for } 8 \text { minutes, pressing the timer button and then } \\
\text { up arrow } 8 \text { times. }\end{array}$ & $\begin{array}{l}\text { 42. Reach for last knob on right and turn to the right until burner } \\
\text { is set to OFF. }\end{array}$ \\
\hline $\begin{array}{l}\text { 16. Walk over and get the measuring cup and bowl from } \\
\text { counter then take measuring cup and bowl to sauce. }\end{array}$ & 43. Put on oven mitts and with both hands. \\
\hline 17. Lift lid and remove from pasta sauce jar. & 44. Lift and carry pot to sink. \\
\hline 18. Using both hands, gently pour into measuring cup until full. & $\begin{array}{l}\text { 45. Carefully pour water and pasta into the strainer in the } \\
\text { sink. }\end{array}$ \\
\hline $\begin{array}{l}\text { 19. Take measuring cup of sauce and pour into microwave safe } \\
\text { bowl without spilling. }\end{array}$ & $\begin{array}{l}\text { 46. Walk back to stove with pot and place it on the left } \\
\text { burner. }\end{array}$ \\
\hline 20. Place lid back on to jar and twist to tighten. & $\begin{array}{l}\text { 47. Take off mitts and place on counter, then walk back to the } \\
\text { sink. }\end{array}$ \\
\hline $\begin{array}{l}\text { 21. With both hands, carry microwave safe bowl over to } \\
\text { microwave and open microwave door. }\end{array}$ & $\begin{array}{l}\text { 48. With both hands, lift strainer and carefully shake out } \\
\text { extra water. }\end{array}$ \\
\hline $\begin{array}{l}\text { 22. With both hands, lift microwave safe bowl and gently } \\
\text { place into microwave. }\end{array}$ & $\begin{array}{l}\text { 49. When all of the water is out, carry strainer with } \\
\text { both hands to kitchen counter where plate and fork are. }\end{array}$ \\
\hline $\begin{array}{l}\text { 23. Locate and walk to the paper towel roll and tear off one } \\
\text { piece of paper towel. }\end{array}$ & $\begin{array}{l}\text { 50. With left hand, hold strainer and with right hand hold fork. Tilt } \\
\text { the strainer over plate and with fork in one hand scrape pasta onto } \\
\text { plate. }\end{array}$ \\
\hline $\begin{array}{l}\text { 24. Place sheet of paper towel on top of microwave safe } \\
\text { bowl. }\end{array}$ & 51. Set strainer down on counter. \\
\hline 25. Close microwave door. & $\begin{array}{l}\text { 52. Pick up bowl of sauce and tilt and pour sauce carefully } \\
\text { over pasta then set the sauce bowl back down on counter. }\end{array}$ \\
\hline 26. Set for 2 minutes by pressing 2-0-0 to start. & 53. With fork, stir pasta and sauce together. \\
\hline 27. While sauce is heated, orient to kitchen stove. & 54. Eat meal. \\
\hline
\end{tabular}

FIgURE 1: Task analysis for making pasta.

[12] note that "interobserver agreement is the most commonly used indicator of measurement quality in applied behavior analysis" (p. 113) and describe it as "the degree to which two or more independent observers report the same observed values after measure the same events" (p. 113). In this study, the researchers used a trial-by-trial interobserver agreement. An independent observer collected data once during the baseline phase and four times during the intervention phase for each participant. The observers' data sheets were then compared to match their scoring of each step of the task analysis. During the baseline phase, the interobserver agreement was $100 \%$. During the intervention phase, the mean interobserver agreement was 99\% (range 90-100\%). Watkins and Pacheco [13] express the concern 


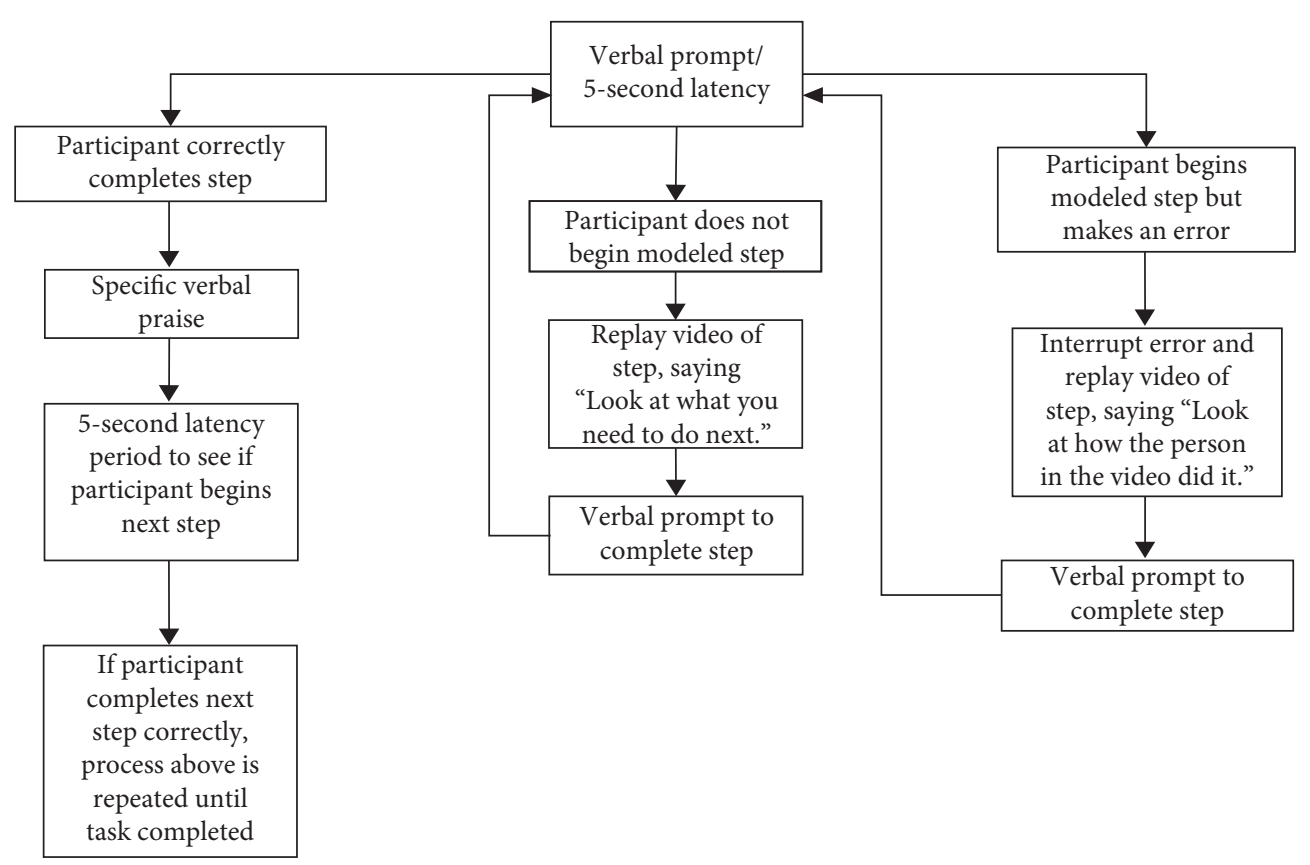

Figure 2: Video modeling intervention procedures.

that "percentage of agreement statistics tend to inflate the degree of perceived observer agreement" (p. 207) and recommend the use of the Kappa Coefficient of Agreement, which ranges from -1 to 1 and factors chance agreement between observers into the estimation of interobserver agreement (p. 209). In this study, the Kappa statistic was calculated to confirm the interobserver agreement calculated by the trial-by-trial method. The mean value of the Kappa Coefficient of Agreement was 0.95 (range 0.82-1), which is described as "excellent agreement" (p. 209), because it represents the level of agreement actually observed in proportion to that which would be expected by chance.

3.4.2. Procedural Reliability. Procedural reliability (treatment fidelity) was measured for each instructor. The instructor was scored based on the number of steps of the instructional protocol that she/he completed accurately. Procedural reliability was computed by dividing the number of instructional steps followed by the total number of instructional steps and then multiplying by 100 . Mean procedural reliability was $98.6 \%$ (range $91-100 \%$ ).

3.5. Social Validity. Social validity was assessed through correspondence with the mothers of the students involved and through direct observation of the students' consumption behaviors. Each of the students' mothers was asked at the beginning of the study to specify a food that she believed it would be helpful, to her and to other caregivers, for her child to learn to prepare with minimal supervision. Each mother specified a food item that was frequently requested by her child as a preferred food. Secondly, each student was invited to consume his/her food item at the end of each session. In all instances, the students ate their food items and indicated that the food was tasty, frequently asking for more.
Additionally, the research staff taste-tested the prepared food items and found them to be both edible and appetizing.

\section{Results}

Figures 3 and 4 show the percentage of task steps each participant completed independently and with prompting during the baseline and acquisition phases of the intervention. All participants demonstrated mastery, defined as completion of $90 \%$ of their individual cooking tasks without prompting, within 12 instructional sessions.

Cody completed exactly two steps (3\%) of his pizza preparation task independently during the three baseline sessions. He reached $90 \%$ after seven training sessions and criterion after nine, completing 95\%, 94\%, and 95\% of the task steps independently in his final three training sessions. In a maintenance probe taken six weeks after training stopped, Cody completed $95 \%$ of the 64-step task chain correctly and independently.

For Zeke, the results were similar. Zeke consistently completed one step of his pasta-cooking task correctly during baseline. His progress, however, was rapid. He exceeded $90 \%$ accuracy in the fifth instructional session and reached criterion after eight sessions. Six weeks after intervention, Zeke completed $98 \%$ of the 54 pasta-cooking steps accurately and independently.

\section{Discussion}

The results of the current study support the findings of previous studies that video modeling is an effective instructional strategy for teaching meal preparation skills to adolescents with moderate or severe disabilities $[4,5,9,10]$. The present study differed from these previous studies, though, in several important ways. First, the tasks were more 

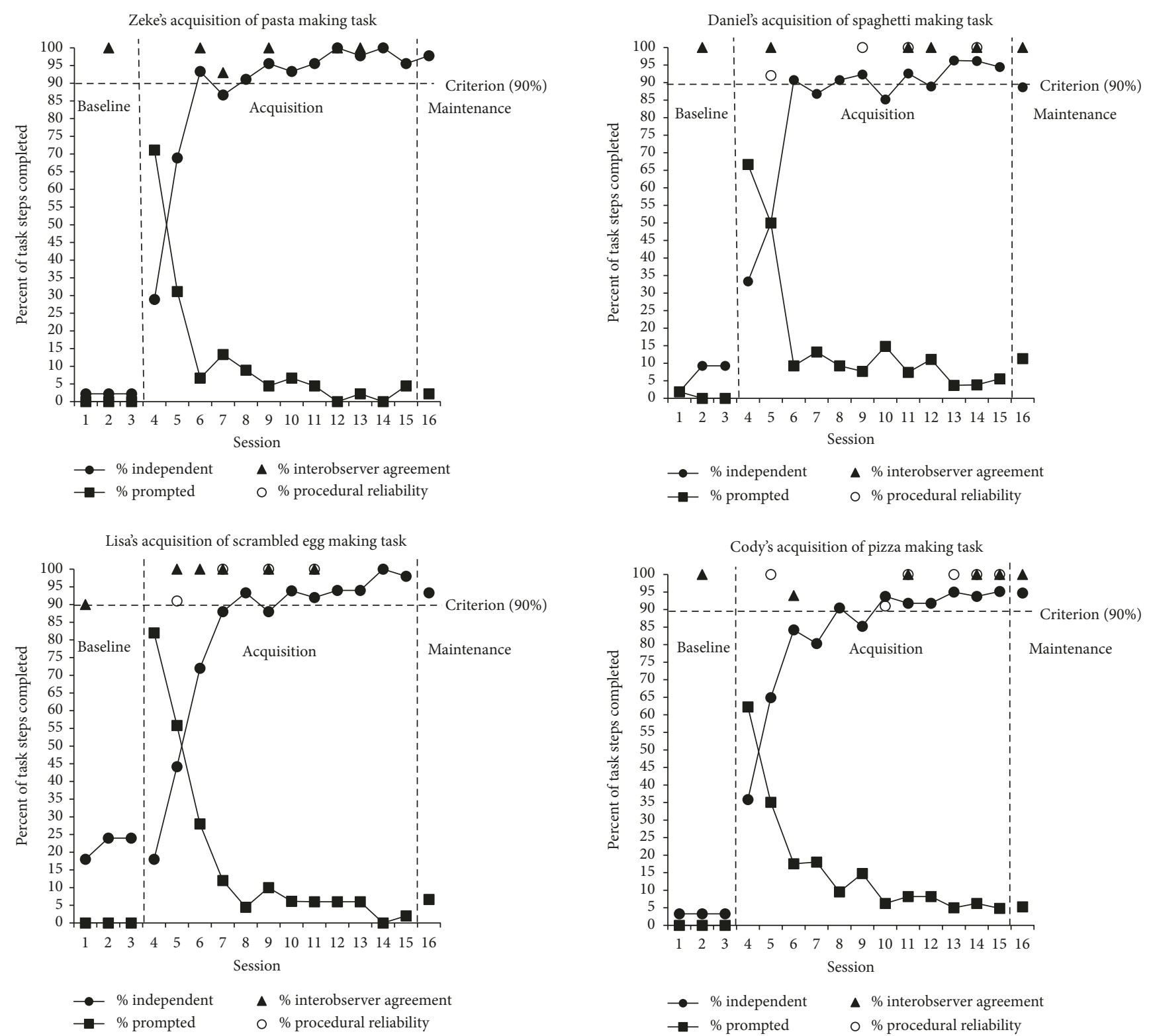

Figure 3: Percentage of task steps completed independently and with prompting: Zeke and Lisa.

complex and involved substantially more steps than the meal preparation tasks taught in the studies discussed previously, such as making a sandwich (12 steps) [9]; completing a math problem (10 steps) [8]; microwaving a frozen pizza or macaroni and cheese or making a smoothie (10-30 steps) [10]; preparing family packs and first aid kits (10 steps each) [14]; and setting a table (12 steps) [11]. Furthermore, in the current study, the pizza-making task also included dishwashing and clean-up components, whereas previous studies did not.

Secondly, this study was also unique in that it involved training the participants "loosely." In their seminal article on generalization procedures, Stokes and Baer [15] define training loosely as "teaching is conducted with relatively little control over the stimuli presented and the correct responses allowed, so as to maximize sampling of relevant

FIGURE 4: Percentage of task steps completed independently and with prompting: Daniel and Cody.

dimensions for transfer to other situations and other forms of the behavior" (p. 357). In the present study, stimuli were randomly varied from session to session. Instead of using the exact same materials in the exact same setting to teach the cooking tasks, different materials, such as different cookware, utensils, and measuring implements, were used. Three of the four videos used for instruction were created in kitchens and using materials different from those found in the Domestic Arts classroom at the school. Moreover, the kitchen workstations at which the students cooked were rotated. The Domestic Arts classroom had four stations.

Students were rotated through these randomly, which presented different physical arrangements for the students to master. Additionally, two of Cody's instructional sessions had to be conducted in his home kitchen. These variations during the acquisition phase of learning are important 
because they may promote the generalization of acquired skills to different settings and different materials. It has been proposed that "high variability in trained tasks enhances cognitive flexibility and results in improved motor performance in novel contexts" [16] (p. 2840). This can lead to generalization [17]. It has been noted that "a therapeutic behavioral change, to be effective, often (not always) must occur over time, persons, and settings, and the effects of the change sometimes should spread to a variety of related behaviors" [15] (p. 350). Simply stated, generalization is necessary for the intervention to have a meaningful and lasting effect on the participants' quality of life.

Due to time limitations, the number of training sessions was restricted and neither maintenance nor generalization phases could be conducted. Nonetheless, the students did demonstrate high levels of skill maintenance six weeks after intervention. Second, because there were only four students involved, the video modeling procedure needs to be replicated with other students and/or in other settings to determine whether the rates of acquisition observed in the current study are representative. Third, the presence of other students cooking in the Domestic Arts room may have introduced incidental learning as an extraneous variable. This is unlikely, however, given that each participant was working on a task that was different from those of his/her peers.

Finally, the intervention included both video modeling and positive reinforcement, in the form of specific verbal praise, for each step completed correctly. It may be difficult to determine the extent to which each component contributed to the participants' acquisition of their assigned cooking task. The video modeling clearly had some effect on the participants' acquisition of the tasks, though, because only the video provided the information needed for the participants to improve their performance, and the replicated pattern and rate of skill acquisition across the four participants suggests that the video modeling was highly effective.

Consistent with results of previous studies, the findings of the current study support the effectiveness of video modeling in teaching meal preparation skills to adolescents with multiple disabilities, including moderate intellectual disabilities. The present study extends the research base on video modeling, however, by focusing on longer, more complex meal preparation tasks in order to promote greater independence, participation, and self-determination for the participants. The rate of skill acquisition observed in the current study is consistent with the rates observed in previous studies $[4,5,9,11]$ and suggests that video modeling may be a very efficient strategy for teaching independent living skills, proving useful for assisting students with disabilities in situations where a life skill needs to be acquired quickly.

Future research is needed to extend the results of this study by implementing video modeling to teach longer and more complex meal preparation tasks (including dishwashing and clean-up skills) and other domestic tasks to individuals of different ages, with different disability types, and in different settings. Nonetheless, the current study adds to the growing body of literature that supports the use of video technologies to enhance independent living opportunities and quality of life for individuals with disabilities.

\section{Data Availability}

The data are available on file at Saint Vincent College.

\section{Conflicts of Interest}

The authors declare that there are no conflicts of interest regarding the publication of this study.

\section{References}

[1] E. Carter, L. Lane, M. Crnobori, A. Bruhn, and W. Oakes, "Self-determination interventions for students with and at risk for emotional and behavioral disorders: mapping the knowledge base," Behavioral Disorders, vol. 36, no. 2, pp. 100-116, 2011

[2] J. W. Madaus, L. Pivarnik, M. Patnoad et al., "Teaching food safety skills to students with disabilities," Teaching Exceptional Children, vol. 42, no. 4, pp. 44-51, 2010.

[3] H. Gable and N. Schoenfeld, "Teaching cooking skills to young women with mild intellectual disability: the effectiveness of Internet websites," Current Issues in Education, vol. 17, no. 1, pp. 1-8, 2014.

[4] L. C. Mechling and M. Gustafson, "Comparison of the effects of static picture and video prompting on completion of cooking related tasks by students with moderate intellectual disabilities," Exceptionality, vol. 17, no. 2, pp. 103-116, 2009.

[5] L. C. Mechling and M. R. Gustafson, "Comparison of static picture and video prompting on the performance of cookingrelated tasks by students with autism," Journal of Special Education Technology, vol. 23, no. 3, pp. 31-45, 2008.

[6] J. Karl, B. C. Collins, K. D. Hager, and M. J. Ault, "Teaching core content embedded in a functional activity to students with moderate intellectual disability using a simultaneous prompting procedure," Education and Training in Autism and Developmental Disabilities, vol. 48, no. 3, pp. 363-378, 2013.

[7] C. A. Miltenberger and M. H. Charlop, "The comparative effectiveness of portable video modeling vs. traditional video modeling interventions with children with autism spectrum disorders," Journal of Developmental and Physical Disabilities, vol. 27, no. 3, pp. 341-358, 2015.

[8] R. O. Kellems and S. Edwards, "Using video modeling and video prompting to teach core academic content to students with learning disabilities," Preventing School Failure: Alternative Education for Children and Youth, vol. 60, no. 3, pp. 207-214, 2015.

[9] M. Al-Salahat, "Using video modeling in teaching a simple meal preparation skill for pupils of Down syndrome," Journal of Education and Practice, vol. 7, no. 9, pp. 82-90, 2016.

[10] J. W. Johnson, E. Blood, A. Freeman, and K. Simmons, "Evaluating the effectiveness of teacher-implemented video prompting on an iPod Touch to teach food-preparation skills to high school students with autism spectrum disorders," Focus on Autism and Other Developmental Disabilities, vol. 28, no. 3, pp. 147-158, 2013.

[11] K. Ayres and D. Cihak, "Computer- and video-based instruction of food-preparation skills: acquisition, generalization, and maintenance," Intellectual and Developmental Disabilities, vol. 48, no. 3, pp. 195-208, 2010. 
[12] J. Cooper, T. Heron, and W. Heward, Applied Behavior Analysis, Pearson Education, Inc, Upper Saddle River, NJ, USA, 2nd edition, 2007.

[13] M. W. Watkins and M. Pacheco, "Interobserver agreement in behavioral research: importance and calculation," Journal of Behavioral Education, vol. 10, no. 4, pp. 205-212, 2000.

[14] D. F. Cihak and L. Schrader, "Does the model matter? Comparing video self-modeling and video adult modeling for task acquisition and maintenance by adolescents with autism spectrum disorders," Journal of Special Education Technology, vol. 23, no. 3, pp. 9-20, 2008.

[15] T. F. Stokes and D. M. Baer, "An implicit technology of generalization1," Journal of Applied Behavior Analysis, vol. 10, no. 2, pp. 349-367, 1977.

[16] J. Adwan-Mansour and T. Bitan, "The effect of stimulus variability on learning and generalization of reading in a novel script," Journal of Speech, Language, and Hearing Research, vol. 60, no. 10, pp. 2840-2851, 2017.

[17] W. X. Yao, W. DeSola, and Z. C. Bi, "Variable practice versus constant practice in the acquisition of wheelchair propulsive speeds," Perceptual and Motor Skills, vol. 109, no. 1, pp. 133-139, 2009. 


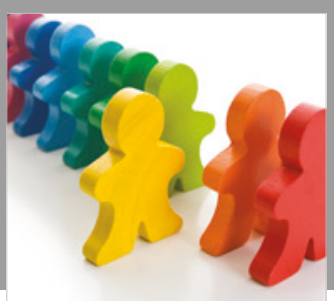

Autism

Research and Treatment
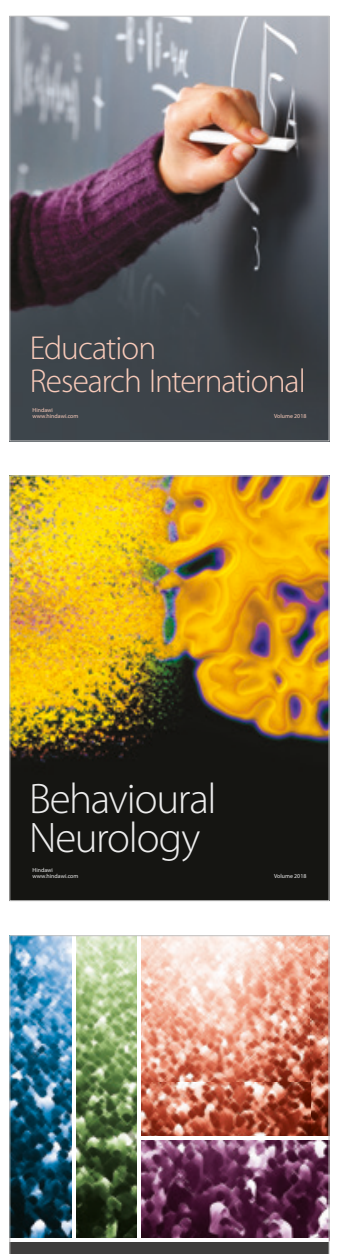

International Journal of

Population Research

$\underline{-m}$

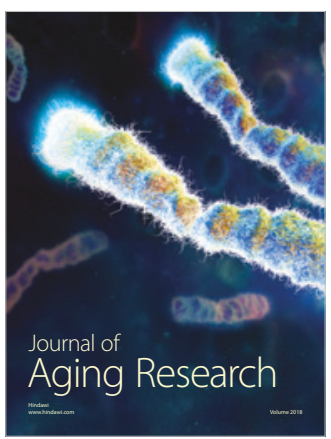

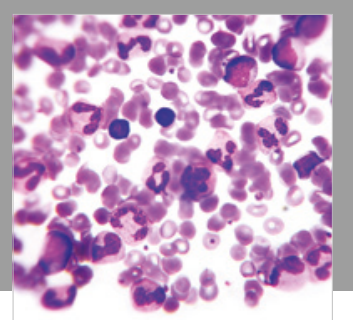

Pathology

Research International$$
=
$$

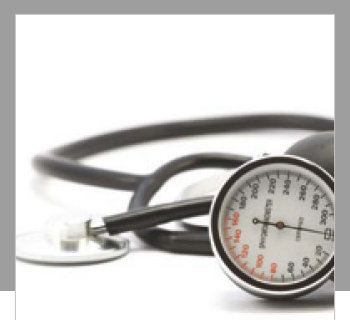

Nursing

Research and Practice

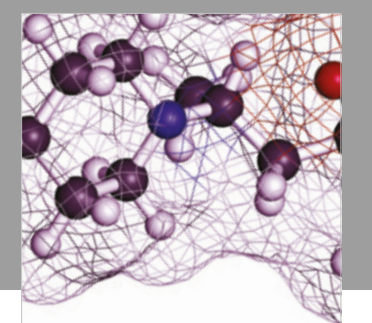

Pain

Research and Management

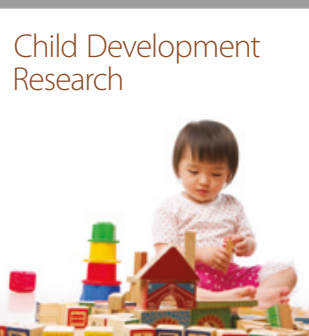

बाD

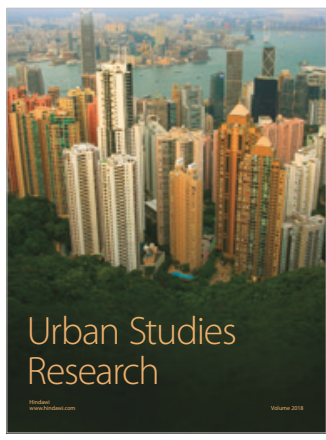

\section{Hindawi}

Submit your manuscripts at

www.hindawi.com
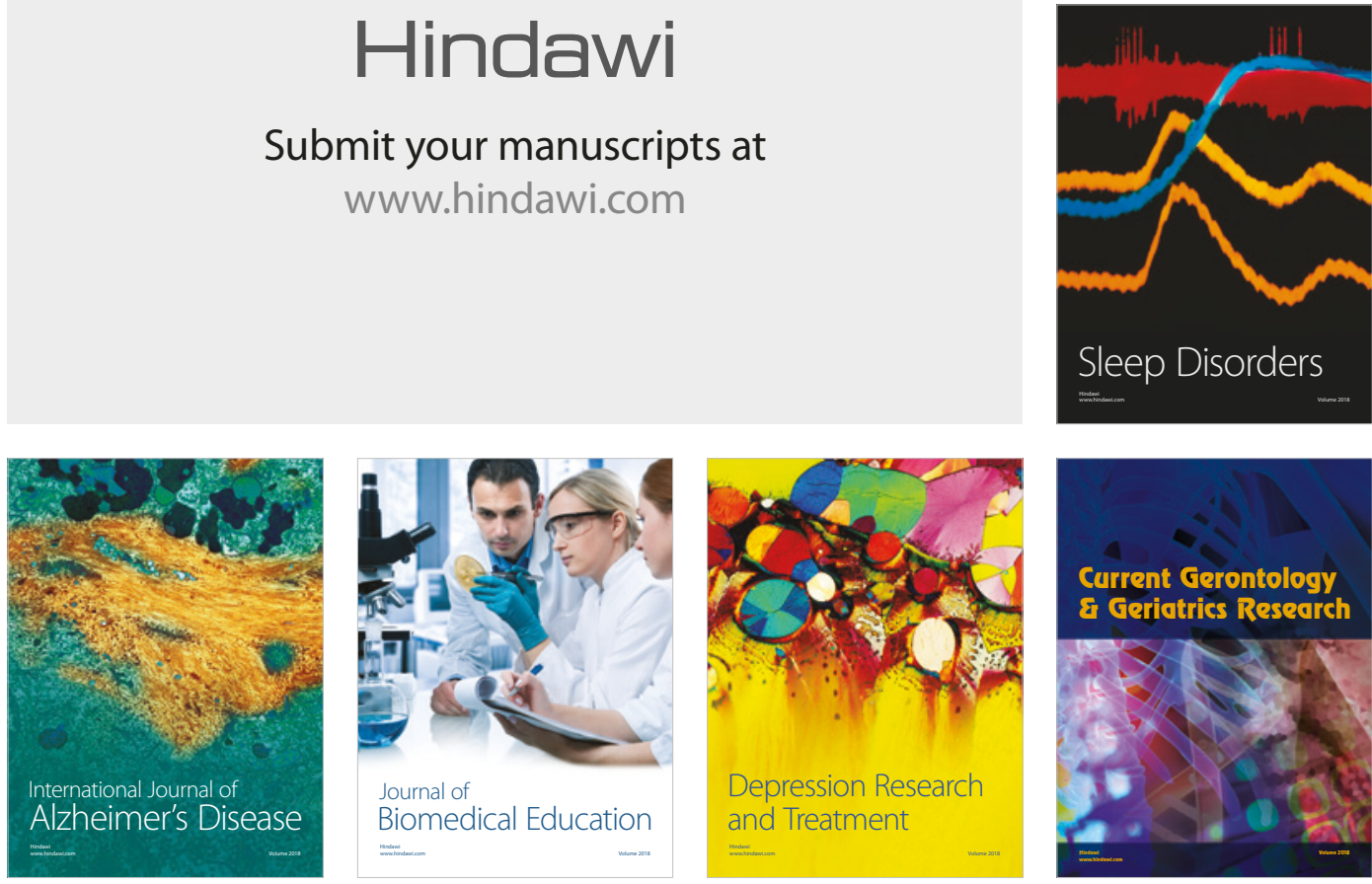

Journal of

Biomedical Education

$=$

smman

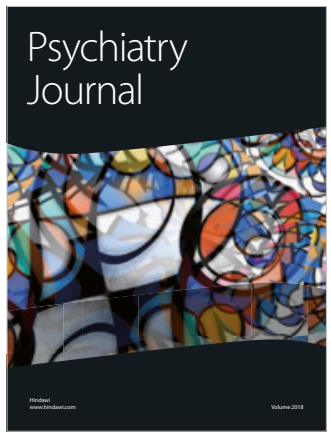

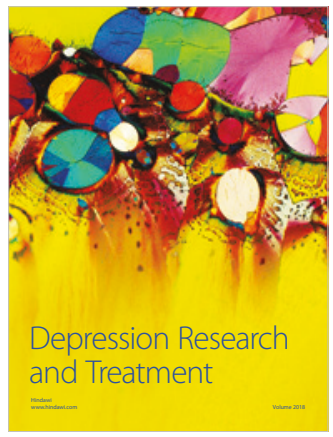
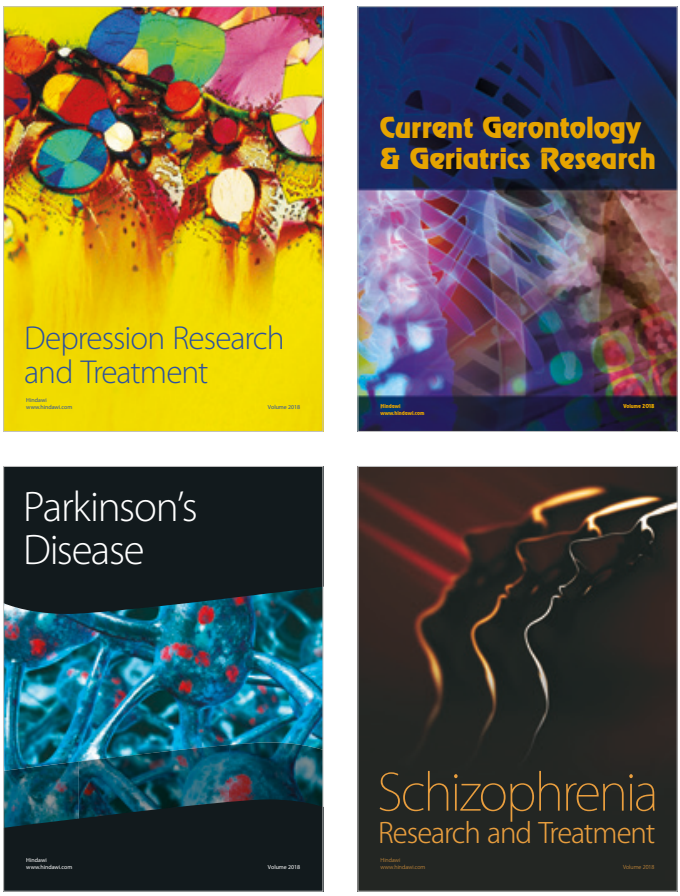